\section{$\underset{\substack{\text { hommes } \\ \text { \& migrations }}}{ }$}

\section{Hommes \& migrations}

Revue française de référence sur les dynamiques

migratoires

$1324 \mid 2019$

Religion et discrimination

\title{
Une prochaine résidence d'écrivain au Musée
}

\section{Marie Poinsot}

\section{OpenEdition \\ Journals}

\section{Édition électronique}

URL : https://journals.openedition.org/hommesmigrations/8956

DOI : 10.4000/hommesmigrations.8956

ISSN : 2262-3353

\section{Éditeur}

Musée national de l'histoire de l'immigration

\section{Édition imprimée}

Date de publication : 1 janvier 2019

Pagination : 178-183

ISBN : 978-2-919040-44-5

ISSN : $1142-852 X$

\section{Référence électronique}

Marie Poinsot, « Une prochaine résidence d'écrivain au Musée », Hommes \& migrations [En ligne], 1324 2019, mis en ligne le 01 janvier 2019, consulté le 06 janvier 2022. URL : http://

journals.openedition.org/hommesmigrations/8956; DOI : https://doi.org/10.4000/

hommesmigrations.8956 


\title{
LHTHERATURE
}

\section{Une prochaine résidence d'écrivain au Musée}

\author{
Marie Poinsot
}

La place de la littérature dans un musée consacré à l'histoire de l'immigration en France s'est construite sur plusieurs années. Cette présence littéraire s'est renforcée grâce à l'organisation des neuf éditions du Prix littéraire de la Porte Dorée, de cafés littéraires, de stages de formation à l'attention des enseignants sur la littérature et l'exil, des deux éditions du salon LittExil et des nombreuses chroniques et entretiens d'auteurs parus dans la revue Hommes \& Migrations.

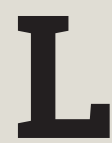

e patrimoine littéraire constitue une ressource essentielle pour retranscrire la densité des situations migratoires, combattre les discours réducteurs sur l'immigration et interroger la faillite de l'hospitalité dans des sociétés européennes qui se referment sur ellesmêmes et semblent renier leurs valeurs humanistes et démocratiques avec la montée des nationalismes de tout poil. Dans cet environnement hostile, les récits littéraires constituent une porte d'entrée privilégiée sur les expériences intimes des migrants, sur leur vulnérabilité, leurs souffrances et leurs angoisses pour certains, mais aussi sur leur soif de découverte et d'émancipation pour ceux qui considèrent l'éloignement des leurs comme la condition d'une vie autonome. Les romans nous laissent entrevoir la profondeur d'horizons humains qui, trop souvent, restent invisibles dans les sociétés d'accueil. « Placée sous le signe de la diversité des récits, la littérature intègre le mouvement jusque dans sa trame narrative. Elle est à l'image des circulations à travers le monde, inventive, innovante, créative dans ses formes et ses jeux de langues et de langages. Elle nous offre une panoplie de ressorts narratifs (reportage, autobiographie, fiction, etc.) et des styles à l'image d'un monde globalisé, interconnecté, en hybridation perpétuelle. Grâce à l'écriture de l'exil, les temporalités, les espaces et les voix humaines s'articulent étroitement pour rendre compte de la complexité des réalités migratoires. Cette littérature vivifie la musicalité de la langue littéraire par tous les apports d'écrivains dont l'adoption du français comme langue d'écriture ne les dispensent pas $d^{\prime} Y$ insuffler leurs mots et une rythmicité nouvelle ${ }^{1}$. 》

1. Marie Poinsot, "Postface», in Paris Ville monde, un regard neuf sur l'immigration, Paris, éd. Rue Saint Ambroise, pp. 209-210. 
Le Musée a voulu insérer dans sa programmation l'accueil d'un écrivain en résidence qui explore les thématiques migratoires en faisant collaborer des publics divers au processus d'écriture. Le Musée a candidaté en 2016 au programme de résidences d'écrivain soutenu par le Conseil régional d'Île-de-France.

\section{Une première résidence centrée sur Paris}

Lors de la parution dans la revue Hommes \& Migrations d'un numéro sur le «Paris des migrants ${ }^{2}$ », Bernardo Toro avait manifesté son intérêt pour traiter l'histoire de l'immigration dans la capitale sous l'angle de la production de nouvelles littéraires. Il pensait que ces récits donneraient à voir différents visages du cosmopolitisme " par le bas » à travers des témoignages de migrants d'horizons divers illustrant les relations qu'ils ont tissées avec un quartier parisien. Il a donc expérimenté en éclaireur ce dispositif de résidence d'écrivain en proposant d'organiser sur l'année 2017 des ateliers de collecte et d'écriture de récits de $v^{2} e^{3}$. La diversité des témoignages reflétait la pluralité des profils de migration, des trajectoires familiales ou personnelles sur Paris. Fidèle à sa démarche participative très ouverte, Bernardo Toro a fait de cette diversité des publics une palette d'une grande richesse. Ainsi, a-t-il ajusté les modalités d'organisation des ateliers d'écriture et de production de textes au degré de maîtrise du français des publics concernés.

Cette résidence a conduit à la parution, en septembre 2018, d'un recueil de nouvelles littéraires, Paris Ville monde, un regard neuf sur l'immigration ${ }^{4}$, aux éditions Rue Saint Ambroise, rattachées à la revue de nouvelles littéraires éponyme. «La plupart des nouvelles qui composent ce recueil sont le fruit d'une rencontre entre un écrivain et un migrant parisien. Le migrant a confié son expérience à un écrivain qui, à partir de son témoignage, a élaboré une fiction qui tantôt retrace fidèlement son parcours, tantôt s'en écarte suivant une logique connue du seul auteur. Afin de saisir l'expérience migratoire dans sa diversité, nous avons convoqué des migrants d'origine et de condition très diverses. On $Y$ retrouvera dix origines différentes, mais aussi divers types d'immigration, des réfugiés politiques aux immigrés économiques, en passant par des expatriés. L'objectif commun de ces textes est de retrouver, par-delà les stéréotypes et les idées reçues, l'expérience singulière du migrant en apportant à son témoignage le plein exercice de la subjectivité. Ces textes, qui explorent des voies très différentes, reposent sur une même conviction: seul le passage à la fiction peut libérer le témoignage de sa mise sous tutelle. La raison principale en est la place toute particulière que la littérature occupe dans nos sociétés. Tout témoin est tenu de répondre de ce qu'il avance, l'écrivain, en revanche, est dégagé de cette responsabilité dans le cadre de sa fiction. Il peut

2. "Paris des migrants », Hommes \& Migrations, $n^{\circ} 1308,2014$.

3. Voir Bernardo Toro, « Comment redonner la parole aux migrants ? Réflexions à mi-parcours sur la résidence d'écriture de Bernardo Toro au Musée national de l'histoire de l'immigration », in Hommes \& Migrations, n 1319, pp. 158-160.

4. Idem. 
rendre publics des propos violents, choquants, ambigus sans avoir à $Y$ répondre à titre personnel. Le pacte fictionnel le protège en lui accordant une liberté que la société refuse aux citoyens ordinaires et a fortiori aux migrants ${ }^{5}$. 》

Il permet également au migrant «témoin 》 de se dégager du poids de son expérience, notamment dans sa dimension traumatique, et de confier son récit à une plume littéraire qui saura le retranscrire par la fiction, en l'enrichissant de cette autre subjectivité, celle de l'auteur, sans pour autant effacer ou corrompre le propos du migrant. "La subjectivité n'est pas un secret que l'écrivain aurait pour mission d'arracher au migrant. Elle n'est pas l'objet d'une délégation. La subjectivité n'apparaît que dans l'espace d'une autre subjectivité pour la simple raison qu'elle n'est pas un contenu, mais un mode de relation entre deux personnes. Essentialiser ce rapport en faisant de la subjectivité un attribut pouvant faire l'objet d'un don, d'un transfert ou d'une restitution serait inadéquat. Une certaine littérature engagée repose pourtant sur cette méprise. En prétendant donner la parole à ceux qui en sont privés, elle les condamne d'autant plus au silence ${ }^{6}$. »

Cette résidence a ainsi permis d'approfondir le processus d'écriture en binôme, un mode particulier de participation des migrants à la production d'une nouvelle littéraire, qui est ainsi contrainte dans sa forme narrative. Elle a permis d'émettre des hypothèses sur la mise en relation entre l'écriture et l'expérience migratoire qui peuvent faire l'objet d'un débat dans les domaines de la création littéraire et de la médiation culturelle, reprises à l'avenir dans le cadre des activités du Musée.

\section{En 2019, une résidence placée sous le signe des exils littéraires}

Le Musée national de l'histoire de l'immigration a été sollicité en juin 2018 par Mohamed Mbougar Sarr pour élaborer un nouveau projet de résidence d'écrivain. Le Musée lui a décerné, en avril 2018, le Prix littéraire de la Porte Dorée pour son ouvrage Le Silence du chœur paru aux éditions Présence Africaine. Le jury avait été unanime sur le choix du lauréat. Proposant de privilégier des romans portant sur des migrations collectives, plutôt que sur des exils individuels traités de manière romantique, il avait déclaré que ce roman était «le livre que nous attendions sur les migrations européennes». Il s'agit en effet d'une épopée collective, avec une dimension épique et un souffle qui est admirablement tenu à travers tout le roman. Le style d'une très belle facture fait varier les registres et permet aux lecteurs d'appréhender tous les personnages dans leur psychologie et leur parcours, que ce soient les migrants africains issus de pays différents et aux destins pris dans leur singularité, mais aussi les habitants siciliens de ce village situé près de l'Etna et qui voient arriver ces migrants, pour certains avec rejet, pour d'autres avec bienveillance et engagement. Jamais caricatural, ni démonstratif, ce livre est l'histoire d'un accueil qui tourne mal, à l'image de l'Europe qui n'est plus habitée par des valeurs d'hospitalité et d'humanisme. La tension monte au fil des pages jusqu'à un

5. Ibid., pp. 7-8.

6. Ibid., pp. 8-9. 
dénouement formidable. À aucun moment, ce jeune écrivain sénégalais, édité pour ce deuxième roman par Présence Africaine, ne pontifie. Il interroge avec maturité tous les comportements des personnages, ceux engagés dans l'accueil ou ceux hostiles et violents, mais aussi les raisons et les logiques des départs de ceux qui sont arrivés. La fin du roman nous plonge dans un cataclysme, annonciateur d'une destinée commune.

Le Musée a ainsi imaginé que Mohamed Mbougar Sarr pouvait accompagner avec force et pertinence les activités littéraires programmées en 2019 en montrant le pouvoir de la littérature pour énoncer les réalités des migrations, au-delà des rumeurs et des discours médiatiques, en cherchant à retranscrire la « densité anthropologique à l'œuvre» (termes de l'auteur). Cette résidence d'écriture sera une occasion formidable de s'adresser de manière privilégiée à des publics du Musée, dans une démarche constructive, participative et valorisante pour eux. Mohamed Mbougar Sarr définit avec précision la manière dont il entend tisser des relations d'écriture avec les publics du Musée. Il envisage de «travailler » avec deux types de publics, avec des logiques de participation et sur des thématiques différentes qui correspondent à leur profil social et à leur relation avec la société française.

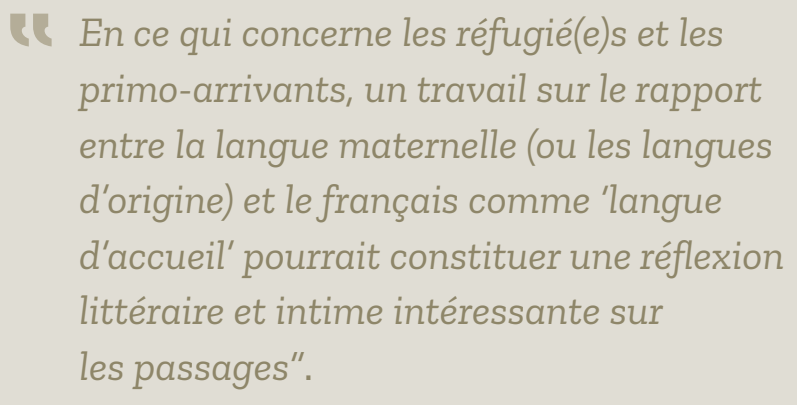

Le premier public avec lequel l'auteur souhaite s'investir est constitué par les migrants récemment arrivés en France. « En ce qui concerne les réfugié(e)s et les primo-arrivants, un travail sur le rapport entre la langue maternelle (ou les langues d'origine) et le français comme "langue d'accueil" pourrait constituer une réflexion littéraire et intime intéressante sur les passages. Passages d'un pays à l'autre, d'un imaginaire à l'autre, mais aussi d'une langue à l'autre, qui rendent compte d'un effort de traduction. Que signifie, qu'implique, intimement, le fait de devoir naviguer d'une langue à l'autre, ou de vivre entre elles ? Comment entrer dans une langue autre? De quelle manière l'apprivoise-t-on ? Comment arriver à exprimer dans celle-ci un imaginaire qui a toujours été lié à une langue différente? Il serait intéressant de voir la façon dont la familiarisation au français, son acquisition progressive, reconfigure la vie intérieure des réfugié(e)s et les oblige à un certain nombre de choix, ruses, stratégies poétiques et "poéthiques" qui sont autant de modalités ou d'étapes d'une identité en mutation. Cette question de la vie entre les langues, de la traduction intime, donc, est d'autant plus essentielle en la matière que les récits de "vécus", "d'histoires", de "trajectoires" sont aussi des aventures linguistiques fortes. Il s'agirait donc d'envisager l'immigration par le biais de la circulation entre les langues, les mots et les émotions que ceux-ci produisent. L'idée de départ de l'atelier serait très simple : demander aux réfugié(e)s qui y participeraient de choisir, chacun et chacune, leur(s) mot(s) préféré(s) dans une de leurs langues d'origine et dans le français, puis tenter un dialogue entre ces mots, c'est-à-dire entre les langues. Peut-être pourra-ton trouver des correspondances, des échos cachés; peut-être découvrira-t-on de 
lointaines origines communes, des recoupements étonnants. En s'appuyant sur l'histoire et la littérature, on pourrait aider les réfugié(e)s, simplement par une attention portée aux mots qui les accompagnent, à mieux nous dire qui ils sont $t^{7}$.»

Par ailleurs, Mohamed Mbougar Sarr souhaite mener des ateliers d'écriture selon d'autres modalités organisationnelles avec des publics de jeunes lycéens. «Le travail avec les lycéens pourrait avoir pour cœur une réflexion sur le sens de l'engagement littéraire. C'est une vieille question, centrale certes - surtout dans le cadre d'un module d'enseignement "littérature et société" -, mais qui a été si traitée qu'il pourrait paraître inutile d'y revenir. Cependant, l'intérêt pourrait résider dans le fait d'aborder autrement cette question de "l'engagement littéraire" : en en faisant une (contre) histoire, bien sûr, mais en essayant surtout de la relativiser, en réfléchissant à la place et la signification de la littérature dans quelques sociétés contemporaines, en questionnant le statut ou la situation des écrivains d'aujourd'hui, en en revenant, en somme, aux pouvoirs ou aux non-pouvoirs de la littérature dans une époque où l'indignation rapide est critiquable (facile, à peu de frais, et souvent systématique) mais où, paradoxalement, les raisons de s'indigner n'ont jamais été si nombreuses. Que signifie dès lors, pour un écrivain, s'engager? Que voudrait dire, par exemple, sur le drame des migrants, s'engager par la littérature? Ces modules pourraient permettre aux lycéens de réfléchir autrement aux catégories "engagement"/"nonengagement" et d'envisager de manière plus complexe le travail de l'écrivain au cœur de sa société. Outre des textes littéraires incontournables (et d'autres, moins connus) sur cette question, on pourrait s'appuyer sur des témoignages d'écrivains invités, issus d'autres cultures, qui ont une manière singulière de penser leur place dans leur société d'origine. L'atelier pourrait avoir pour objectif l'écriture, pour chaque classe, d'un article critique sur la notion d'engagement. Le travail préparatoire à la rédaction de ce texte serait aussi un moyen d'aider les lycéens dans leur évaluation des textes littéraires dans le cadre du Prix littéraire de la Porte Dorée 20198. ॥

La résidence d'écrivain permettra au Musée d'expérimenter d'autres modalités de participation des publics à des activités d'apprentissage de la lecture de textes littéraires et d'écriture littéraire. Cette expérience peut conduire le Musée à poursuivre sa réflexion sur les innovations en matière de mobilisation de démarches inclusives des publics, mais aussi à réfléchir à la manière dont le Musée peut valoriser et diffuser les résultats d'une activité littéraire comme des ressources originales faisant aussi œuvre de mémoires, de témoignages et de patrimoines. Le Musée est ainsi convaincu que cette nouvelle expérimentation peut favoriser une réflexion extrêmement dynamique sur la créativité sur les territoires d'expériences et sur les capacités à faire vivre et entendre auprès d'autres publics des récits de migration particuliers. La résidence devrait conduire à la réalisation des récits de vie avec ces deux types de publics en vue d'une parution dans la revue Hommes \& Migrations et d'une diffusion numérique la plus large possible.

7. Mohamed Mbougar Sarr, dossier de candidature pour la résidence d'écrivain 2019 soutenue par le Conseil régional d'île-de-France.

8. Idem. 
L'accueil de Mohamed Mbougar Sarr pour une résidence d'écriture au Musée lui offre un environnement pertinent pour l'écriture de son prochain roman où il sera plus directement question de trajectoires littéraires. «L'histoire de la littérature est jalonnée "d'exils littéraires", c'est-à-dire de cas d'écrivains qui, pour différentes raisons, partent, disparaissent, se cachent, disent adieu à la littérature ou à l'écriture publique. Le cas de Rimbaud est le plus connu, mais savez-vous l'histoire de Yambo Ouologuem (1940-2017), ce Malien qui a publié en 1968, à vingthuit ans, un brillant premier roman, Le Devoir de violence, couronné par le Prix Renaudot, avant d'être accusé de plagiat et de se retirer de la vie publique, s'enfermant dans un silence amer, et changeant complètement de vie (il est devenu un exégète du Coran) ? C'est un roman non pas sur, mais à partir de son histoire que j'aimerais écrire. Que signifie, pour un écrivain, se taire? Que signifie "partir" lorsqu'on écrit? Pour quelles raisons choisit-on de s'effacer alors qu'on a de toute évidence du talent, voire du génie littéraire? Et, surtout, qu'implique le silence littéraire pour un écrivain? N'est-ce pas là, le silence et la solitude, les conditions véritables d'une œuvre artistique authentique, délivrée des pressions, modes et attentes de la vie littéraire publique? L'histoire de Yambo Ouologuem me fascine. Elle me semble être une métaphore de la quête de silence que tout écrivain cherche peut-être, et qui est l'une de mes obsessions. Je voudrais consacrer un livre à cette question, celle des écrivains qui fuient, partent, disparaissent, se déplacent. Le roman raconterait l'histoire d'une jeune femme, universitaire, fascinée par un écrivain dont elle ne sait presque rien, et qui n'a publié que deux petits livres. Cette jeune femme chercherait le secret de cette œuvre mystérieuse. Elle chercherait aussi sa propre vérité. Et, naturellement, sa quête littéraire et existentielle serait aussi une enquête, pour savoir ce qu'est devenu l'écrivain qui la fascine, lequel a disparu un demi-siècle plus tôt, sans laisser de traces, pour d'obscures raisons ${ }^{9}$. »

Mohamed Mbougar Sarr souhaite être un écrivain très présent au Musée sur l'ensemble de son année de résidence. La présence d'un tel écrivain apportera en retour une dynamique très profitable aux équipes du musée en suscitant des échanges, des questionnements, des implications de l'équipe à la résidence d'écrivain. L'auteur pourra, par exemple, intégrer le comité de lecture interne du Musée qui sélectionne les romans pour la liste nominative du Prix littéraire de la Porte Dorée en 2019, puis être membre du jury comme cela s'est toujours fait depuis le début. Cette nouvelle résidence s'inscrit dans le cadre de la préparation des 10 ans du Prix littéraire du Palais de la Porte Dorée qui donnera lieu à une programmation ambitieuse autour de la littérature de l'exil au printemps prochain. À partir d'avril 2019, l'écrivain souhaite organiser des «conversations » avec d'autres écrivains dans le cadre de la programmation littéraire de la médiathèque. Ces réflexions sur les exils littéraires seront ainsi discutées entre pairs et débattues également grâce à un échange toujours convivial avec le public du Musée. 